\title{
Facilitadores e barreiras no desenvolvimento da gestão ambiental em instituições de educação superior: análise dos relatórios de sustentabilidade da Global Reporting Initiative
}

\author{
Enablers and barriers to environmental management \\ development in higher education institutions: an analysis \\ of sustainability reports of Global Reporting Initiative \\ Maicom Sergio Brandao ${ }^{1 *}\left(\mathbb{0}\right.$, Aldo Roberto Ometto ${ }^{\circledR}$, Patrícia Cristina Silva Leme ${ }^{1}(0$, \\ Victor Eduardo Lima Ranieri ${ }^{2} \mathbb{1}$, Marinho Gomes de Andrade Filho ${ }^{2}$
}

\begin{abstract}
RESUMO
O objetivo deste trabalho é identificar quais características das instituições de educação superior (IES) atuam como facilitadoras ou barreiras no desenvolvimento de ações de gestão ambiental. A análise de regressão beta de 38 relatórios de sustentabilidade elaborados conforme as diretrizes da Global Reporting Initiative (GRI) mostrou que a facilidade de acesso a recursos financeiros, a existência de plano ou política ambiental/ de sustentabilidade e a natureza pública da instituição podem ser consideradas facilitadoras para o desenvolvimento da gestão ambiental em IES, enquanto a alta complexidade da estrutura administrativa e a existência de diversos campi podem ser entendidas como barreiras para seu desenvolvimento. O estudo auxilia tomadores de decisão a entender como as particularidades das IES e o contexto em que se inserem impactam no desenvolvimento das ações de gestão ambiental.

Palavras-chave: instituições de educação superior; gestão ambiental; relatórios de sustentabilidade.
\end{abstract}

\begin{abstract}
This research aims at exploring which characteristics of Higher Education Institutions act as enablers or barriers in the development of environmental management actions. The beta regression analysis of 38 sustainability reports prepared in accordance with the Global Reporting Initiative (GRI) guidelines showed that the institution's ease of access to financial resources, the existence of an environmental/sustainability plan or policy and the public nature can be considered as enablers for the development of environmental management in $\mathrm{HEl}$, while high complexity of the administrative structure and the existence of several campuses can be understood as barriers to its development. The study helps decision makers understand how the particularities of HEls and the context in which they operate impact the development of environmental management actions
\end{abstract}

Keywords: higher education institutions; environmental management: sustainability reports.

\section{INTRODUÇÃO}

A sustentabilidade é o desafio global para as sociedades deste milênio. Reverter os danos ambientais causados pelo modelo de desenvolvimento atual dos países tem sido uma tarefa complexa, principalmente pelos conflitos de interesse que contrapõem, de um lado, as demandas econômicas e, de outro, as sociais e ambientais. Para promover o desenvolvimento economicamente próspero, socialmente justo e ambientalmente adequado, são necessários esforços coletivos e individuais, públicos e privados.

Como partícipe da sociedade e referência de conhecimento, as instituições de educação superior (IES) desempenham pelo menos dois papéis na concepção dos caminhos que guiam as sociedades para a sustentabilidade: o primeiro deles consiste em sua contribuição para o processo educativo para a formação de pessoas e o segundo como 
o de instituição exemplar e memorável em gestão ambiental de seus campi (LEITE et al., 2014).

Para isso, as IES têm avançado na inclusão da sustentabilidade na pauta das discussões que envolvem o planejamento de currículos e o desenvolvimento de seus campi, ampliando, assim, a inserção do tema nas esferas de tomada de decisão. Como suporte ao processo de integração, diversas IES têm criado um órgão que trate especificamente dos conteúdos de sustentabilidade e/ou gestão ambiental dentro do organograma institucional.

No entanto, ainda restam muitos desafios. Apesar de ter-se verificado certo avanço ao longo das últimas décadas, a inserção da temática da sustentabilidade de forma transversal aos quatro eixos da universidade - i.e., pesquisa, ensino, extensão e gestão - requer, quase sempre, uma mudança no modo padrão de compreender o mundo e depara com barreiras como as do racionalismo e do reducionismo das questões ambientais para temas de interesse predominantemente econômico e de mercado (SIBBEL, 2009).

Dentro do âmbito da gestão universitária, a temática ambiental, que é componente de uma das dimensões da sustentabilidade - seguindo a conceituação de Sachs (1993) —, tem sido gradativamente inclusa nas estruturas institucionais das universidades seja por meios usualmente mais informais, como os círculos de debates e discussões, seja por meios programáticos, em que há o entendimento e a formalização do compromisso com o meio ambiente e as dinâmicas ambientais tangíveis às IES.

Considerando o desenvolvimento histórico da temática ambiental nas universidades, as primeiras manifestações para a inserção da temática do desenvolvimento sustentável nas IES datam do início da década de 1970 com a Declaração de Estocolmo. No entanto, é a partir dos anos 90, com a Declaração de Talloires, que essa discussão torna-se mais evidente e se iniciam os programas para a denominada "ambientalização" da universidade (ROORDA, 2001; WRIGHT, 2002; ALSHUWAIKHAT; ABUBAKAR, 2008), que pode ser entendida como a inserção da "dimensão socioambiental onde ela não existe ou é tratada de forma inadequada" (KITZMANN, 2007, p. 554). Como resultado, ações e iniciativas têm sido desenvolvidas nas últimas décadas para inserir a temática da sustentabilidade nos diversos eixos sustentadores da universidade.

Em relação ao eixo da gestão, diversos autores destacam o compromisso das universidades no desenvolvimento de sistemas de gestão ambiental nos campi universitários para que elas possam ser instituições de referência no tratamento das questões ambientais para a sociedade em geral (CLUGSTON; CALDER, 1999; NICOLAIDES, 2006; TAUCHEN; BRANDLI, 2006; HIDALGO; DEL ÁLAMO, 2007; CARAMEZ; COOPER, 2011).

Para isso, pode se entender que o primeiro passo consiste em estruturar o planejamento institucional voltado à sustentabilidade para que o campus possa desenvolver ações estruturadas e programas relacionados fundamentalmente à atuação da correção e à prevenção de impactos (HIDALGO; DEL ÁLAMO, 2007), atuando, por exemplo, por meio da gestão sustentável da água, da energia e dos espaços verdes e propondo caminhos que reduzam as emissões de gases estufa em especial, reduzindo a Pegada Ecológica (CLUGSTON; CALDER, 1999; UHL; ANDERSON, 2001).

No entanto, a gestão ambiental em campi universitários não é limitada somente ao atendimento de aspectos relacionados à água, à energia e aos espaços verdes. As IES também devem estabelecer diretrizes dentro de seus planejamentos que contemplem sistemas de compras baseados em critérios socioambientais e programas de reutilização e tratamento de insumos, tais como materiais de escritório e reagentes químicos, além de buscar estratégias de redução do consumo de papel por meio da gestão digital da informação ou ações que incentivem o uso de meios de transporte não motorizados (WRIGHT, 2002; ALSHUWAIKHAT; ABUBAKAR, 2008; KAPLAN, 2015).

Velazquez et al. (2006) definem que uma IES que deseje alcançar a sustentabilidade deve promover, dentro das esferas regional ou global, a minimização dos efeitos negativos ambientais, econômicos, sociais e de saúde que possam ocorrer pela utilização dos recursos nas atividades de ensino, pesquisa, gestão e extensão, servindo como apoio à sociedade na transição de estilos de vida sustentáveis.

Como forma de operacionalizar a proposta de um campus sustentável, diversos autores têm mobilizado esforços para a criação de modelos de referência para a gestão ambiental em IES. Viebahn (2002) estrutura um sistema de gestão ambiental que contempla ações para oito aspectos das universidades, a saber: estrutura organizacional, diretrizes ambientais, regulamentações externas, auditorias ambientais, objetivos e metas ambientais, relatórios e sistemas de informações ambientais, a própria educação ambiental e o envolvimento da comunidade.

Posteriormente, Savely, Carson e Delclos (2007) estruturaram um modelo de gestão ambiental para universidades e colégios dos Estados Unidos com base nos fundamentos de Veibahn (2002) e no sistema de gestão ambiental da ISO 14001. Dentre os vários elementos propostos pelos autores, destacam-se a presença da política ambiental; o desenvolvimento de programas com objetivos e metas; a manutenção de documentação e relatórios de desempenho ambiental; as necessidades de procedimentos internos que orientem as práticas de gestão ambiental etc.

Cabe reforçar que os autores apresentam modelos que consideram os aspectos regionais de IES sem propor modelos universais, sendo condizentes com a argumentação de Corcoran, Walker e Wals (2004), os quais abordam a sustentabilidade como um tema sensível aos contextos e às realidades locais, e de Uhl e Anderson (2001), que destacam o respeito pelos aspectos naturais, econômicos e culturais de cada região.

No entanto, ainda existem diversos desafios de diferentes naturezas para que seja desenvolvido um sistema de gestão ambiental que abranja todos os temas da sustentabilidade em IES. Esses desafios variam desde a estruturação teórico-conceitual do sistema de gestão ambiental (LEITE 
et al., 2014) até os aspectos mais práticos, como o levantamento e a destinação de recursos financeiros (VAGNONI; CAVICCHI, 2015), lidando também com os possíveis entraves à mudança por parte da comunidade universitária (VELAZQUEZ; MUNGUIA; SANCHEZ, 2005; TOO; BAJRACHARYA, 2015).

Além dos desafios, acredita-se que existam fatores próprios, característicos e até intrínsecos das IES que contribuam de forma positiva ou negativa para o desenvolvimento de ações e iniciativas em gestão ambiental (VELAZQUEZ; MUNGUIA; SANCHEZ, 2005). O tamanho da comunidade universitária tem sido estudado recentemente como um potencial fator que está associado à facilidade de captação de recursos para o financiamento ambiental nos campi (LARRÁN; ANDRADES, 2015). Da mesma forma acontece com órgãos e estruturas de gestão que centralizem as decisões e tracem caminhos da instituição com o meio ambiente (CLUGSTON; CALDER, 1999).

Tendo como base o cenário apresentado, o objetivo deste trabalho é identificar fatores próprios e característicos das IES que contribuam de forma positiva ou negativa para o avanço da gestão ambiental nesse tipo de instituição.

A identificação de fatores próprios dessas instituições que possam afetar as ações ambientais pode contribuir para aprimorar o processo de tomada de decisão pelos gestores, facilitando o entendimento sobre os fatores que potencializam ou limitam o desenvolvimento de ações ambientais no contexto em que atuam.

\section{METODOLOGIA}

A pesquisa pode ser considerada de abordagem quantitativa e de caráter exploratório por buscar evidências do nível de desenvolvimento da gestão ambiental em IES com base na avaliação de relatórios de sustentabilidade. Entende-se que a análise dos resultados de um processo avaliativo - no caso, relatórios de sustentabilidade certificados - forneça condições adequadas para identificar as principais ações de gestão ambiental dentro das IES, podendo ser utilizados como fonte de dados para estudos, pois representam uma fonte certificada de declaração da instituição sobre seu desempenho.

\section{Fonte de dados e amostra}

Os dados analisados nesta pesquisa foram extraídos de relatórios de sustentabilidade elaborados por IES. Foram utilizados os relatórios de sustentabilidade que estavam disponíveis na base de dados da Global Reporting Initiative (GRI). A GRI é a estrutura mais conhecida para a construção voluntária de relatórios de sustentabilidade (BROWN; DE JONG; LEVY, 2009). Farneti e Guthrie (2009) identificaram que a essa iniciativa é uma opção para a elaboração de relatórios de sustentabilidade para diversas instituições, inclusive públicas, as quais, segundo Lozano (2011), necessitam desenvolver melhor a prática de elaboração de relatórios de sustentabilidade, considerando o baixo número de relatórios existentes para esse tipo de instituição.

A estrutura da GRI é composta de indicadores dentro de temas relacionados à sustentabilidade. Em relação à dimensão ambiental, a terceira geração (GRI-G3) apresenta o conjunto de 30 indicadores ambientais distribuídos em 9 temas - água, energia, materiais, emissões, biodiversidade, produtos e serviços, conformidade, transporte e geral (GRI, 2012).

A pesquisa abrange o período de 2008 a 2013, correspondente à terceira geração de relatórios GRI - G3. No momento da coleta de dados, o número total de relatórios existentes da base de dados era de 135 , sendo que, desse conjunto, a quantidade inicial selecionada para estudo foi de 89 relatórios, $66 \%$ do total. Posteriormente, o número de relatórios foi reduzido a 38 para aplicação de um modelo de regressão (Quadro 1).

Dentro da primeira seleção de relatórios de sustentabilidade, 46 relatórios não foram analisados devido aos seguintes motivos: indisponibilidade para download via base de dados da GRI; escrita em outra língua que não fosse português, inglês ou espanhol; ou ausência do conteúdo referente à dimensão ambiental da sustentabilidade, que é o foco deste trabalho.

Em relação à distribuição das instituições analisadas por país, verifica-se a predominância de Espanha, Chile e Estados Unidos, que, juntos, representam $58 \%$ do total de instituições. Em seguida, o Brasil, com 4 IES, representa 10\%, seguido da Austrália (3 IES). O conjunto dos outros países da amostra - Colômbia, Suíça, Suécia, Canadá, China etc. - compõem os $24 \%$ restantes.

\section{Procedimento para extração e análise dos dados}

As IES elaboram seus relatórios segundo as diretrizes da GRI, mas não seguem necessariamente um padrão para sistematização dos indicadores reportados, isto é, a maioria delas reporta os indicadores ao longo dos textos de seus relatórios sem construir um resumo daqueles pontuados no relatório. Dessa maneira, o processo de extração dos dados realizou-se por meio da leitura, na qual foram identificados os indicadores reportados no corpo dos textos dos 89 relatórios considerados na etapa inicial.

A sistematização dessas informações extraídas foi feita com base na atribuição do valor 0 para situações em que o indicador não estava presente no relatório da IES ou 1 para quando o indicador fora relatado parcialmente ou totalmente pela instituição. Cada um desses valores foi documentado em uma tabela que associava os 89 relatórios analisados com os 30 indicadores de sustentabilidade ambiental propostos pela GRI, gerando um conjunto de dados com 2.670 observações.

Uma vez construída a tabela com as observações de presenças e ausências dos indicadores nos relatórios, criou-se um índice para caracterizar o nível de aplicação da GRI pelas IES. O nível de aplicação é um conceito utilizado pela própria GRI para atribuir uma pontuação final para o relatório creditado. No entanto, para este estudo, o índice criado 
difere relativamente do proposto pela GRI, pois apresenta caráter exclusivamente quantitativo, isto é, considera somente a proporção de indicadores relatados em relação ao conjunto total de 30 indicadores, diferentemente do que ocorre pela GRI, que contempla outros requisitos.

De forma geral, a fórmula do índice proposto é dada pela Equação 1:

$\mathrm{I}_{\mathrm{IES} j}^{\mathrm{i}}=\sum_{\mathrm{n}}^{1} \mathrm{a} /\left(\sum_{\mathrm{n}}^{1} \mathrm{a}+\sum_{\mathrm{n}}^{1} \mathrm{~b}\right)$

Quadro 1 - Conjunto de instituições de ensino superior selecionadas para o estudo agrupadas por país.

\begin{tabular}{|c|c|}
\hline Universidade & País \\
\hline Deakin University & Austrália \\
\hline La Trobe University & Austrália \\
\hline Monash University & Austrália \\
\hline Hogeschool-Universiteit Brussel (HUB) & Bélgica \\
\hline Anhanguera Educacional & Brasil \\
\hline Fundação Dom Cabral & Brasil \\
\hline Instituto Superior de Administração e Economia & Brasil \\
\hline Universidade Feevale & Brasil \\
\hline University of Calgary & Canadá \\
\hline Pontifica Universidad Católica de Valparaíso & Chile \\
\hline Universidad de Santiago do Chile & Chile \\
\hline Universidad del Bio-Bio & Chile \\
\hline University of Hong Kong & China \\
\hline Universidad Tecnológica de Bolivar & Colômbia \\
\hline ESADE Foundation & Espanha \\
\hline Escuela Técnica Superior de Ingenieros Ind. UPM & Espanha \\
\hline Florida Centre de Formació & Espanha \\
\hline Universidad de Cádiz & Espanha \\
\hline Universidad de Cantabria & Espanha \\
\hline Universidad de Zaragoza & Espanha \\
\hline Universidad Internacional de Andalucía & Espanha \\
\hline Universidad Nacional de Educación a Distancia & Espanha \\
\hline Universidade de Santiago de Compostela & Espanha \\
\hline Ball State University & Estados Unidos \\
\hline Brown University & Estados Unidos \\
\hline Michigan State University & Estados Unidos \\
\hline North Carolina State University & Estados Unidos \\
\hline Pomona College & Estados Unidos \\
\hline University of California - Berkeley & Estados Unidos \\
\hline University of Massachusetts -Dartmouth & Estados Unidos \\
\hline University of Michigan & Estados Unidos \\
\hline University of Texas - Arlington & Estados Unidos \\
\hline Villanova University & Estados Unidos \\
\hline Aalto University & Finlândia \\
\hline Universidade do Minho & Portugal \\
\hline University of Gothenburg & Suécia \\
\hline École Polytechnique Fédérale de Lausanne & Suíça \\
\hline ETH Zurich & Suíça \\
\hline
\end{tabular}

Em que:

$\mathrm{I}_{\mathrm{IES}}^{\mathrm{i}}=$ relatório analisado considerando o sub-índice $j$ como indicação da IES relatora e $i$ como o ano do relatório;

$n=$ número de relatórios avaliados;

$a=$ presenças identificadas dos indicadores ambientais da GRI no relatório da IES;

$b=$ ausências de indicadores no relatório.

O índice proposto, mais do que revelar a proporção de aplicação da GRI, associa-se à abrangência e ao desenvolvimento da gestão ambiental dentro da IES, porque traduz ações em uma medida quantificável, considerando o envolvimento ou não das IES com os temas e indicadores estabelecidos pela GRI.

A escolha dos fatores avaliados como facilitadores ou barreiras para o desenvolvimento da gestão ambiental em IES ocorreu por meio de buscas realizadas na literatura associada ao tema da gestão e educação ambiental em IES e também considerando fatores adicionais elencados pelos autores (TAUCHEN; BRANDLI; FRANDOLOSO, 2010; LARRÁN; ANDRADES, 2015). A partir dos resultados da busca, identificaram-se aqueles que pudessem ser coletados por meio da leitura dos relatórios de sustentabilidade das IES. Os fatores selecionados são apresentados no Quadro 2.

Quadro 2 - Fatores próprios das instituições de ensino superior avaliados no estudo.

\begin{tabular}{|c|c|c|c|}
\hline Código & $\begin{array}{c}\text { Fator } \\
\text { identificado }\end{array}$ & Dado atribuído & Fonte \\
\hline \multirow{3}{*}{1} & \multirow{3}{*}{ Tamanho da IES } & Número de alunos & \multirow{3}{*}{$\begin{array}{l}\text { Larrán e } \\
\text { Andrades (2015) } \\
\text { e Ferrer-Balas } \\
\text { et al. (2008) }\end{array}$} \\
\hline & & Número de funcionários & \\
\hline & & Números de docentes & \\
\hline 2 & Idade da IES & $\begin{array}{l}\text { Número de anos de } \\
\text { existência }\end{array}$ & $\begin{array}{c}\text { Escolhido pelos } \\
\text { autores }\end{array}$ \\
\hline 3 & $\begin{array}{c}\text { Órgão } \\
\text { ambiental ou de } \\
\text { sustentabilidade }\end{array}$ & $\begin{array}{l}\text { Existência de órgão } \\
\text { institucional de gestão } \\
\text { ambiental ou de } \\
\text { sustentabilidade }\end{array}$ & $\begin{array}{l}\text { Clugston e } \\
\text { Calder (1999) }\end{array}$ \\
\hline 4 & $\begin{array}{l}\text { Política/plano } \\
\text { ambiental }\end{array}$ & $\begin{array}{l}\text { Presença de política } \\
\text { ambiental/de } \\
\text { sustentabilidade, plano } \\
\text { estratégico ambiental/ } \\
\text { de sustentabilidade ou } \\
\text { conteúdo ecológico/ } \\
\text { de sustentabilidade em } \\
\text { plano estratégico }\end{array}$ & $\begin{array}{c}\text { Velazquez, } \\
\text { Munguia e } \\
\text { Sanchez (2005), } \\
\text { Savely, Carson e } \\
\text { Delclos (2007), } \\
\text { Sorrentino, } \\
\text { Nascimento e } \\
\text { Portugal (2011) }\end{array}$ \\
\hline 5 & Natureza da IES & IES pública ou privada & $\begin{array}{c}\text { Larrán e } \\
\text { Andrades (2015) }\end{array}$ \\
\hline 6 & $\begin{array}{l}\text { Certificação } \\
\text { ambiental }\end{array}$ & $\begin{array}{l}\text { Existência de certificação } \\
\text { de conteúdo ambiental }\end{array}$ & $\begin{array}{l}\text { Ruscheinsky e } \\
\text { Gomes (2015) }\end{array}$ \\
\hline 7 & $\begin{array}{c}\text { Centralização } \\
\text { versus } \\
\text { descentralização } \\
\text { territorial e } \\
\text { administrativa }\end{array}$ & $\begin{array}{l}\text { Número de campi } \\
\text { segregados fisicamente } \\
\text { pertencentes às IES }\end{array}$ & $\begin{array}{c}\text { Escolhido pelos } \\
\text { autores }\end{array}$ \\
\hline
\end{tabular}

IES: instituição de ensino superior. 
Os fatores apresentados no Quadro 2 foram estabelecidos pelo potencial que apresentam de facilitar ou limitar o desenvolvimento da gestão ambiental nos campi das IES, conforme indicado pela literatura. Para identificar como esses fatores relacionam-se com o indicador criado, elaborou-se um modelo de regressão beta devido às particularidades do conjunto de dados e objetivo da pesquisa.

A regressão beta proposta por Ferrari e Cribari-Neto (2004) abrange casos em que a variável de interesse em uma pesquisa é limitada pelo intervalo $(0,1)$. Para a aplicação, os autores propõem uma parametrização alternativa da densidade beta que pode ser identificada na Equação 2:

$\left.\mathrm{f}(\mathrm{y} ; \mu, \varphi)=(\Gamma(\varphi) / \Gamma(\mathrm{y} \varphi) \Gamma((1-\mathrm{y}) \varphi)) \mathrm{y}^{\mathrm{y} \varphi-1}(1-\mathrm{y})^{(1-\mu) \varphi-1}\right)$

$0<\mathrm{y}<1$, em que $0<\mu<1$ e $\varphi>0$. Escrevendo y $\sim \mathrm{B}(\mu, \varphi)$, em que $\mathrm{E}(\mathrm{y})=\mu$ e VAR $(\mathrm{y})=\mu(1-\mu) / 1+\varphi$. O parâmetro $\varphi$ é conhecido como parâmetro de precisão desde que, fixado $\mu$, quanto maior $\varphi$, menor a variância de y (FERRARI; CRIBARI-NETO, 2004). Fazendo $y_{1}, \ldots, y_{n}$ variáveis aleatórias independentes, em que cada $y_{t}, t=1, \ldots, n$ segue a densidade beta, com média $\mu_{t}$ e precisão $\varphi$. O modelo de regressão obtido pode ser definido pela Equação 3:

$\mathrm{g}(\mu \mathrm{t})=\sum^{\mathrm{k}} \mathrm{x}_{\mathrm{ti}} \beta_{\mathrm{i}}=\mathrm{n}_{\mathrm{t}}$

Em que:

$\beta=\left(\beta_{1}, \ldots, \beta_{k}\right)^{\mathrm{T}}=$ vetor de parâmetros de regressão desconhecidos $\left(\beta \in \mathrm{R}^{\mathrm{K}}\right)$;

$\mathrm{x}_{\mathrm{t} 1}, \ldots, \mathrm{x}_{\mathrm{tk}}=$ observações de $\mathrm{k}$ covariáveis $(\mathrm{k}<\mathrm{n})$, que são assumidas como fixas e conhecidas.

Para função de ligação de $\mathrm{g}(\cdot)$, adotou-se a logit, dada pela Equação 4:

$\mu_{t}=\frac{e^{x^{\mathrm{T}}} \beta}{1+e^{\mathrm{x}_{t}^{\mathrm{T}}} \beta}$

Em que:

$\mathrm{x}_{\mathrm{t}}^{\mathrm{T}}=\left(\mathrm{x}_{\mathrm{t} 1}, \ldots, \mathrm{x}_{\mathrm{tk}}\right), \mathrm{t}=1, \ldots, \mathrm{n}$.

Apesar de o conjunto de dados levantados por meio do cálculo do índice de nível de aplicação da GRI satisfazer as condições para aplicação do modelo de regressão, percebeu-se a necessidade de se realizar um filtro para incluir somente um relatório por IES, porque se entende que o uso de diversos relatórios de uma mesma IES poderia enviesar a análise, ponderando os resultados para aquelas instituições que apresentassem maior quantidade de relatórios elaborados dentro do intervalo de tempo estudado (2008-2013). Para isso, definiu-se que, para as IES que tivessem mais de um relatório no conjunto inicial de 89 relatórios analisados (que representava aproximadamente $70 \%$ das observações), seria selecionado para a análise de correlação o relatório de sustentabilidade que apresentasse o maior valor do índice de nível de aplicação.

Para os casos de único relatório que abrangesse mais de um ano, isto é, bianual ou cujo ano fiscal da IES abrangesse dois anos, definiu-se o ano mais recente como ano do relatório. Essa escolha não alterou a consistência da análise, pois o aspecto de diferença entre anos não foi analisado. Por fim, o segundo filtro limitou a análise a um conjunto de 38 relatórios/instituições para os quais foi aplicado o modelo de regressão beta.

\section{RESULTADOS}

A análise dos dados forneceu o conjunto de resultados que são apresentados nesta seção. A Tabela 1 revela o resultado do cálculo do índice correspondente ao nível de aplicação da GRI - abrangência e desenvolvimento dos temas ambientais nos campi para cada uma das IES avaliadas. Os dados indicam que, quanto mais próximo de 1 , maior é a quantidade de indicadores reportados pelas IES e, consequentemente, tende a existir maior diversidade de indicadores ambientais explorados pelas IES dentro de seus programas de gestão ambiental. No caso do estudo, os índices variaram de 0,1 (Estados Unidos) até 0,999 (Estados Unidos e Austrália), o que indica a presença de todos os indicadores ambientais propostos na GRI no relatório de sustentabilidade elaborado.

A Tabela 2 contempla as medidas descritivas do conjunto de dados. Em relação ao número de alunos, o valor médio por IES foi de 30.967 . A presença de alta variação dentro do conjunto de dados é revelada pelo valor calculado do coeficiente de variação e pelos valores máximo e mínimo da amostra. Para as quantidades de docentes (média de 2.699) e funcionários (média de 2.235) das IES, ocorre situação similar, com alta variação dos dados refletida pelo coeficiente de variação e pelos valores mínimo e máximo.

O número médio de anos de existência das IES é de 101 anos, com variação entre 2 e 518 anos. $\mathrm{O}$ valor da mediana indica a presença de assimetria positiva da distribuição dos dados, podendo-se entender que aproximadamente $50 \%$ das IES têm até 54 anos de existência. Finalmente, em relação ao número de campi, o valor médio é de 2,47 , com valores mínimo de 1 campus e máximo de 6 campi.

Considerando a porcentagem de IES de natureza pública ou privada, $78,95 \%$ são públicas, enquanto apenas $21,05 \%$ fazem parte do grupo de IES privadas. Em 97,37\% das IES do conjunto avaliado, há a presença de política ambiental ou de sustentabilidade, plano estratégico ambiental/de sustentabilidade ou conteúdo ecológico/de sustentabilidade como componente de seu plano estratégico.

No entanto, a presença de estruturas organizacionais para suporte ao desenvolvimento da temática ambiental ou de sustentabilidade na IES não acompanhou a presença de declarações ou compromissos 
Tabela 1 - Índice de aplicação da Global Reporting Initiative para o conjunto final de relatórios analisados no estudo.

\begin{tabular}{|c|c|c|c|}
\hline Relatório/IES & País de origem da IES & Ano & $\mathrm{I}_{\mathrm{IESj}}$ \\
\hline 1 & Austrália & 2013 & 0,467 \\
\hline 2 & Austrália & 2013 & 0,533 \\
\hline 3 & Austrália & 2011 & 0,999 \\
\hline 4 & Bélgica & 2013 & 0,467 \\
\hline 5 & Brasil & 2010 & 0,167 \\
\hline 6 & Brasil & 2011 & 0,233 \\
\hline 7 & Brasil & 2013 & 0,267 \\
\hline 8 & Brasil & 2013 & 0,433 \\
\hline 9 & Canadá & 2013 & 0,367 \\
\hline 10 & Chile & 2010 & 0,500 \\
\hline 11 & Chile & 2012 & 0,600 \\
\hline 12 & Chile & 2012 & 0,633 \\
\hline 13 & China & 2011 & 0,533 \\
\hline 14 & Colômbia & 2009 & 0,300 \\
\hline 15 & Espanha & 2008 & 0,133 \\
\hline 16 & Espanha & 2011 & 0,167 \\
\hline 17 & Espanha & 2010 & 0,233 \\
\hline 18 & Espanha & 2011 & 0,267 \\
\hline 19 & Espanha & 2011 & 0,267 \\
\hline 20 & Espanha & 2009 & 0,333 \\
\hline 21 & Espanha & 2013 & 0,433 \\
\hline 22 & Espanha & 2011 & 0,567 \\
\hline 23 & Espanha & 2013 & 0,567 \\
\hline 24 & Estados Unidos & 2013 & 0,100 \\
\hline 25 & Estados Unidos & 2013 & 0,333 \\
\hline 26 & Estados Unidos & 2011 & 0,367 \\
\hline 27 & Estados Unidos & 2013 & 0,367 \\
\hline 28 & Estados Unidos & 2013 & 0,400 \\
\hline 29 & Estados Unidos & 2010 & 0,467 \\
\hline 30 & Estados Unidos & 2013 & 0,567 \\
\hline 31 & Estados Unidos & 2013 & 0,600 \\
\hline 32 & Estados Unidos & 2011 & 0,900 \\
\hline 33 & Estados Unidos & 2011 & 0,999 \\
\hline 34 & Finlândia & 2012 & 0,433 \\
\hline 35 & Portugal & 2011 & 0,367 \\
\hline 36 & Suécia & 2009 & 0,267 \\
\hline 37 & Suíça & 2009 & 0,333 \\
\hline 38 & Suíça & 2013 & 0,700 \\
\hline
\end{tabular}

IES: instituição de ensino superior. ambientais elaborados pelas IES, pois somente 60,53\% das instituições apontaram a existência de um órgão próprio para o gerenciamento das ações de sustentabilidade. De forma similar, a existência de certificação ambiental foi encontrada em $68,42 \%$ das instituições avaliadas.

Por fim, a Tabela 3 resume os resultados do melhor modelo criado com base na análise de regressão beta. Os fatores que não constam na tabela não foram incluídos por não se mostrarem estatisticamente relevantes.

O conjunto de dados mostra que dos nove fatores avaliados, cinco apresentaram algum tipo de significância em relação aos valores do índice de nível de aplicação da GRI. Entretanto, número de anos de existência da IES, presença de órgão institucional responsável pela área ambiental/de sustentabilidade, número de docentes e certificação ambiental foram fatores para os quais não se encontraram evidências que justificassem suas presenças no modelo de regressão elaborado.

Dois dos fatores identificados como relevantes apresentaram sinal positivo - número de alunos e presença de política ou plano ambiental/de sustentabilidade - , indicando associação positiva com o índice. Os demais revelaram associação negativa - número de funcionários, natureza da IES e número de campi. Somente para o caso do fator que caracteriza a natureza da instituição, para adequação procedimental do método escolhido, o sinal negativo foi atribuído às IES públicas, pois foi atribuído valor zero para elas e 1 para as IES privadas.

Tabela 3 - Resultados do melhor ajuste do modelo de regressão.

\begin{tabular}{l|c|c|c|c} 
& Valor & $\begin{array}{c}\text { Erro } \\
\text { padrão }\end{array}$ & Z valor & $\operatorname{Pr}(>|z|)$ \\
$\begin{array}{l}\text { Número de } \\
\text { alunos }\left(\beta_{\text {alunos }}\right)\end{array}$ & $3,29 \mathrm{E}-05$ & $1,54 \mathrm{E}-05$ & 2,146 & $>0,050$ \\
\hline $\begin{array}{l}\text { Número de } \\
\text { funcionários }\left(\boldsymbol{\beta}_{\text {funcionários }}\right)\end{array}$ & $-1,10 \mathrm{E}-04$ & $5,45 \mathrm{E}-05$ & $-2,009$ & $>0,050$ \\
\hline $\begin{array}{l}\text { Presença de política } \\
\text { ou plano ambiental/ } \\
\text { de sustentabilidade } \\
\left(\boldsymbol{\beta}_{\text {politicaplano }}\right)\end{array}$ & $1,18 \mathrm{E}+01$ & $5,87 \mathrm{E}+00$ & 2,004 & $>0,050$ \\
\hline $\begin{array}{l}\text { Natureza pública ou } \\
\text { privada da IES }\left(\boldsymbol{\beta}_{\text {natureza }}\right)\end{array}$ & $-1,2 \mathrm{OE}+00$ & $4,54 \mathrm{E}-01$ & $-2,640$ & $>0,010$ \\
\hline $\begin{array}{l}\text { Número de } \\
\text { campi }\left(\boldsymbol{\beta}_{\text {campi }}\right)\end{array}$ & $-4,77 \mathrm{E}-01$ & $1,35 \mathrm{E}-01$ & $-3,534$ & $>0,001$ \\
\hline
\end{tabular}

IES: instituição de ensino superior.

Tabela 2 - Resumo descritivo do conjunto dos relatórios analisados na análise de regressão.

\begin{tabular}{l|c|c|c|c|c|c} 
& Média & Desvio padrão & CV & Mínima & Máxima & Mediana \\
\hline Número de alunos & 30.967 & 62.951 & 2,03 & 1.584 & 400.000 & 18.965 \\
\hline Número de funcionários & 2.699 & 3.692 & 1,36 & 98 & 18.986 & 1.489 \\
\hline Número de docentes & 2.235 & 2.675 & 1,19 & 6 & 12.176 & 1.368 \\
\hline Número de anos & 101,55 & 112,83 & 1,11 & 2 & 518 & 54 \\
\hline Número de campi & 2,47 & 1,35 & 0,546 & 1 & 6 & 2 \\
\hline
\end{tabular}

CV: coeficiente de variação. 


\section{DISCUSSÃO}

Os próximos tópicos associam os valores encontrados por meio da aplicação do modelo de regressão construído com base nas informações dos relatórios de sustentabilidade das IES com as evidências textuais encontradas nos relatórios analisados e com os pontos observados por outros autores na literatura, reforçando e expandindo visões sobre os fatores facilitadores e as barreiras para a implantação e o desenvolvimento da gestão ambiental em IES.

\section{Tamanho da comunidade: financiamento e complexidade da estrutura administrativa}

Em relação ao tamanho da comunidade, as quantidades de alunos e de funcionários apresentaram-se como fatores relevantes para a explicação dos índices de nível de aplicação da estrutura de relatórios da GRI. O sinal positivo revelado pelo coeficiente representante dos alunos $\left(\beta_{\text {alunos }}=+3.29 \mathrm{E}-05, \mathrm{p}<0,05\right)$ indica que existe relacionamento positivo entre os parâmetros analisados, sendo assim, maior quantidade de alunos indica maior valor no índice de nível de aplicação da GRI.

Por sua vez, o sinal negativo do fator atribuído ao número de funcionários $\left(\beta_{\text {funcionários }}=-1.10 \mathrm{E}-04, \mathrm{p}<0,05\right)$ reflete efeito inverso ao que ocorre com o número de alunos, indicando que menores números dos primeiros estão associados com maiores valores dos índices de nível de aplicação dos indicadores ambientais da GRI pelas IES.

O tamanho da comunidade da universidade e a relação com o desenvolvimento de ações ambientais e de sustentabilidade têm sido estudados recentemente por um grupo de autores. Ferrer-Balas et al. (2008) atribuem ao tamanho da universidade um papel duplo e não necessariamente convergente quando se refere a inovações em sustentabilidade. Por um lado, os autores defendem que instituições com mais de 10.000-12.000 alunos tendem a apresentar uma estrutura complexa e difícil transformação. Por outro lado, sugerem que as IES menores podem ter dificuldade em obter recursos financeiros e, por isso, limitar o avanço de ações em sustentabilidade.

Com base na literatura sobre o tema (VELAZQUEZ; MUNGUIA; SANCHEZ, 2005; VAGNONI; CAVICCHI, 2015; LO, 2015), nos relatórios analisados e nos resultados deste estudo, verificou-se que, para o grupo de instituições analisadas, o tamanho da comunidade de alunos pode ser considerado um fator diretamente associado ao levantamento de recursos financeiros destinados ao desenvolvimento de ações em sustentabilidade ambiental, sendo que instituições de menor porte, i.e. número de alunos menor que 12 mil, teriam mais dificuldades para levantamento de recursos financeiros em comparação às maiores.

Em relação ao número de funcionários, Velazquez, Munguia e Sanchez (2005) parecem trazer uma contribuição que auxilia na explicação do valor negativo encontrado. Os autores afirmam que a estrutura organizacional pode ser vista como um fator que impacta no desenvolvimento das ações em sustentabilidade. Entende-se que existe associação entre menores quantidades de funcionários e estruturas menos hierarquizadas, compostas de menos cargos e funções, facilitando a tomada de decisão mais rápida. Dessa forma, o sentido dos resultados obtidos parece caminhar para essa direção.

Por fim, os resultados obtidos por meio desta pesquisa não contradizem o que propõe Ferrer-Balas et al. (2008), mas sugerem que, para o pilar da gestão ambiental, IES com maiores quantidades de alunos tendem a ter mais facilidade na aquisição de recursos financeiros para investimento em tecnologias e capacitação de pessoal sobre procedimentos de gestão para o uso racional dos recursos naturais. Além disso, há indícios de que quantidades menores de funcionários podem estar associadas a menores níveis de complexidade da estrutura administrativa.

\section{Política/plano institucional ambiental ou de sustentabilidade}

Os resultados mostraram que existe uma relação positiva e expressiva entre a presença de política ou plano ambiental ou de sustentabilidade $\left(\beta_{\text {politica/plano }}=+1.18 \mathrm{E}+01\right.$ e $\left.\mathrm{p}<0,05\right)$ e maiores índices do nível de aplicação dos indicadores ambientais da GRI pelas IES.

A necessidade do desenvolvimento de planos ou políticas ambientais/de sustentabilidade para IES é apontada por diversos autores (e.g., LEITE et al., 2014; SORRENTINO; BIASOLI, 2014) como forma de direcionar o conjunto de iniciativas ou ações socioambientais na pesquisa, no ensino, na extensão e na gestão que já estão em curso nas IES, mas que ocorrem de forma isolada e pontual.

As políticas/planos são ferramentas que auxiliam a criação de metas e diretrizes para a incorporação programática da temática ambiental dentro dos processos de gestão institucional ou mesmo na própria reflexão sobre o papel e os caminhos que a IES deve tomar em direção à sustentabilidade (VELAZQUEZ; MUNGUIA; SANCHEZ, 2005; SORRENTINO; NASCIMENTO; PORTUGAL, 2011; LEITE et al., 2014).

Em relação ao conjunto de instituições analisadas, verificou-se que mais de $97 \%$ apresentavam algum tipo de política ou plano ambiental associado às ações ambientais e de sustentabilidade desenvolvidas e que existiam diversos motivadores para a formulação desses compromissos.

A institucionalização e o agrupamento de práticas e esforços em sustentabilidade que já ocorrem nos âmbitos de atuação da IES e a formalização do comprometimento com as questões ambientais foram identificados como motivadores para a formulação de planos/políticas ambientais/de sustentabilidade para um grupo de instituições (MOREIRA et al., 2014). Outro ponto observado em diversos relatórios foi a necessidade de cumprimento de exigências legais de órgãos reguladores governamentais (CARAMEZ; COOPER, 2011). Identificou-se também o caso em que os compromissos firmados internacionalmente contribuíram para a formulação das políticas de sustentabilidade. Destacam-se, como exemplos de compromissos, as Declarações de Estocolmo, de Tbilisi, de Talloires, entre outras (WRIGHT, 2002). 
Por fim, o retorno financeiro associado ao investimento em melhorias das práticas ambientais, com melhoria da eficiência de processos internos, foi outro aspecto apresentado como motivador para a elaboração de políticas. No entanto, apesar da real contribuição à melhoria dos processos internos em relação ao uso dos recursos, Velazquez, Munguia e Sanchez (2005) alertam que o reducionismo da problemática ambiental a uma proposta individualista e mercantil agrava a despolitização e as mudanças profundas que se espera nos paradigmas da sociedade na transição para um modo de vida sustentável.

Em resumo, a presença de políticas para a sustentabilidade tem se mostrado uma realidade em diversas IES, e o presente estudo revelou indícios de que a sua formulação pode ser vista como uma prática desejável para avanços na dimensão ambiental/ecológica da sustentabilidade nessas instituições, pois estão associados aos índices mais altos de uso dos indicadores analisados.

As políticas formalizam, em diversos casos, os esforços que estão em curso nas IES e, em outros, possibilitam o surgimento de novas iniciativas. As declarações internacionais sobre meio ambiente que estão relacionadas a universidades e outras instituições de ensino têm se mostrado um fator que favorece o desenvolvimento das políticas de sustentabilidade.

\section{Naturezas pública ou privada da IES}

O conjunto de dados indicou que existe associação entre a natureza da IES e o nível de aplicação dos indicadores ambientais da GRI. O valor encontrado para índice esteve associado com IES de natureza pública $\left(\beta_{\text {natureza }}=-1,20 \mathrm{E}+00\right.$ e $\left.\mathrm{p}<0,01\right)$. O sinal negativo é o que faz referência a esse tipo de instituição, pois, durante a extração das informações, atribuiu-se o valor 0 para as instituições de natureza pública e 1 para as privadas, conforme descrito nas seções anteriores.

Dessa forma, os resultados mostraram que existe maior associação entre valores mais altos do nível de aplicação da GRI e IES públicas. Considerando as instituições analisadas, o conceito de público está atrelado ao recebimento de repasses governamentais, podendo ou não receber contribuições financeiras adicionais provenientes de outras esferas ou cobranças. Considerando os países de origem das instituições analisadas, pelo menos $60 \%$ compartilham desse entendimento para a natureza pública da instituição.

Por depender, em partes, do repasse de recursos do governo, a limitação de repasses financeiros é uma barreira comum compartilhada pelas IES públicas, o que torna o gerenciamento dos recursos financeiros um fator crítico para a manutenção e expansão das estruturas e o desenvolvimento em sustentabilidade. Além disso, outras características contribuem para a explicação dos resultados obtidos, como a necessidade de prestação de contas das operações das IES para a sociedade, obrigações com a legislação para que sejam produzidos relatórios (SAVELY; CARSON; DELCLOS, 2007; FARNETI; GUTHRIE, 2009) ou mesmo alinhamento entre políticas ambientais de instâncias maiores de poder, para atender às pressões de órgãos públicos e legislações nacionais (CARAMEZ; COOPER, 2011).

\section{Centralização versus descentralização territorial e administrativa}

Conforme os resultados da pesquisa, identificou-se que existe associação negativa entre o valor do índice de nível de aplicação da GRI e o número de campi de uma IES $\left(\beta_{\text {campi }}=-4,77 \mathrm{E}-01\right.$ e $\left.\mathrm{p}<0,001\right)$.

$\mathrm{O}$ resultado apresentado revela indícios do impacto da dispersão espacial e administrativa dos campi das IES para o desenvolvimento de ações de sustentabilidade no âmbito da gestão ambiental. Shriberg (2002) aponta um exemplo em que a descentralização atua como um limitante para o avanço de uma tomada de decisão mais dinâmica sobre questões de meio ambiente e sustentabilidade e defende que a descentralização coloca nesse jogo uma série de atores que possuem interesses, não necessariamente convergentes, que fragilizam o desenvolvimento de ações em sustentabilidade. Da mesma forma, Moreira et al. (2014) apontam que a estrutura fragmentada representa uma condição desfavorável em processos de formulação e estabelecimento de políticas integradas de gestão de recursos naturais ou resíduos.

Outro ponto associado com a descentralização espacial e administrativa é o que se refere às especificidades da IES. Corcoran, Walker e Wals (2004) apontam que o tema da sustentabilidade é sensível aos contextos e às realidades locais da instituição. Por essa razão, as ações planejadas em sustentabilidade para determinada universidade atendem de forma específica à realidade da própria instituição, podendo ser complicada a expansão dessas ações para outras realidades.

Para os relatórios analisados, identificou-se um caso em que a presença de diversos campi de uma instituição representou claramente uma barreira para a implantação de ações ambientais precursoras a um plano de sustentabilidade.

\section{CONCLUSÃO}

Este estudo avaliou as características das IES que atuam como facilitadoras ou limitantes para o desenvolvimento de ações em gestão ambiental.

Os achados apontam que o tamanho da comunidade, definido pela quantidade de alunos, pode ser um facilitador para a gestão ambiental, considerando que uma maior quantidade de alunos pode estar associada a uma maior facilidade em obter recursos financeiros. Por sua vez, o tamanho da comunidade, quando visto pelo número de funcionários, pode representar um limitante quando a estrutura administrativa e organizacional torna-se complexa e dificulta os fluxos e a rapidez da tomada de decisão.

Também foi identificado que a presença de política ambiental/de sustentabilidade é desejável para o desenvolvimento da gestão ambiental em IES, pois ela integra, direciona e planeja esforços institucionais. 
Ademais, a natureza pública das instituições está associada a maiores valores do indicador de nível de aplicação da GRI. Nesse caso, entende-se que o compromisso pelo bom uso dos recursos públicos repassados pelas estruturas de governos do Estado e o cumprimento com a legislação podem contribuir como motivadores para o desenvolvimento da gestão ambiental nas IES.

O número de campi de uma instituição também foi considerado significativo, e o estudo revelou que menos campi são preferíveis para o desenvolvimento da gestão ambiental nas IES. Entende-se que menos campi lidem com menor complexidade de integração e sincronismos entre áreas e instâncias pertencentes a uma mesma instituição e que apresentem menos pontos de divergências de natureza regional na gestão para a sustentabilidade.
Uma das limitações do estudo consiste na adoção da GRI como ferramenta de avaliação da sustentabilidade que, apesar de ser uma estrutura que tem sido adaptada pelas IES, não teve como objetivo primário atender a esse tipo de instituição. Outro ponto consiste no fato de que os dados da pesquisa foram coletados por meio da leitura de documentos/relatórios e não pelo contato direto com as instituições estudadas, o que pode ter restringido discussões mais profundas, mas, para o estudo, permitiu atingir os objetivos propostos.

Por fim, entende-se que o estudo avançou ao complementar e expandir o conhecimento científico sobre o desenvolvimento da gestão ambiental em IES. Ele auxilia tomadores de decisão a entender como as particularidades dessas instituições e o contexto em que se inserem impactam no desenvolvimento de ações de gestão ambiental.

\section{REFERÊNCIAS}

ALSHUWAIKHAT, H.M.; ABUBAKAR, I. (2008) An integrated approach to achieving campus sustainability: assessment of the current campus environmental management practices. Journal of Cleaner Production, v. 16, n. 16, p. 1777-1785. https://doi.org/10.1016/j.jclepro.2007.12.002

BROWN, H.S.; DE JONG, M.; LEVY, D.L. (2009) Building institutions based on information disclosure: lessons from GRl's sustainability reporting. Journal of Cleaner Production, v. 17, n. 6, p. 571-580. https://doi.org/10.1016/j.jclepro.2008.12.009

CARAMEZ, R.; COOPER, M. (2011) Plano diretor para a gestão ambiental universitária: a experiência do campus "Luiz de Queiroz" da universidade de São Paulo (USP). In: LEME, P.C.S.; PAVESI, A.; ALBA, D.H.; DIAZ, M.J.G. (orgs.). Visões e experiências ibero-americanas de sustentabilidade nas universidades. Granada: Gráficas Alhambra. v. 1. p. 97-102.

CLUGSTON, R.M.; CALDER, W. (1999) Critical dimensions of sustainability in higher education. Sustainability and University Life, v. 5, p. $31-46$

CORCORAN, P.B.; WALKER, K.E.; WALS, A.E. (2004) Case studies, make-your-case studies, and case stories: a critique of case-study methodology in sustainability in higher education. Environmental Education Research, v. 10, n. 1, p. 7-21. http://doi. org/10.1080/1350462032000173670

FARNETI, F;; GUTHRIE, J. (2009) Sustainability reporting by Australian public sector organisations: Why they report. In: ACCOUNTING FORUM. Anais... Elsevier. p. 89-98.

FERRARI, S.; CRIBARI-NETO, F. (2004) Beta regression for modeling rates and proportions. Journal of Applied Statistics, v. 31, n. 7 , p. 799-815. https://doi.org/10.1080/0266476042000214501

FERRER-BALAS, D.; ADACHI, J.; BANAS, S.; DAVIDSON, C.I.; HOSHIKOSHI, A.; MISHRA, A.; MOTODOA, Y.; ONGA, M.; OSTWALD,
M. (2008) An international comparative analysis of sustainability transformation across seven universities. International Journal of Sustainability in Higher Education, v. 9, n. 3, p. 295-316. https://doi. org/10.1108/14676370810885907

GLOBAL REPORTING INITIATIVE (GRI). (2012) What is GRI? Disponível em: <https://www.globalreporting.org/Information/ about-gri/Pages/default.aspx>. Acesso em: 10 fev. 2012.

HIDALGO, D.A.; DEL ÁlAMO, J.B. (2007) Análisis de los procesos de gestión y educación para la sostenibilidad en las universidades públicas españolas. In: DOCTORADO INTERUNIVERSITARIO EN EDUCACIÓN AMBIENTAL (org.). Tendencias de la Investigación en Educación Ambiental al desarrollo socioeducativo y comunitario. Espanha: Naturaleza y Parques Nacionales. p. 197-215

KAPLAN, D.H. (2015) Transportation sustainability on a university campus. International Journal of Sustainability in Higher Education, v. 16, n. 2, p. 173-186. https://doi.org/10.1108/IJSHE-03-2013-0023

KITZMANN, D. (2007) Ambientalização de espaços educativos: aproximações conceituais e metodológicas. Revista eletrônica do mestrado em Educação Ambiental, v.18, p. 553-574.

KITZMANN, D.I.S.; ANELLO, L.F.S. (2014) Da política pública à política ambiental: a emergência da sustentabilidade nos sistemas universitários federais. In: RUSCHEINSKY, A.; GUERRA, A.F.S. FIGUEIREDO, M.L.; LEME, P.C.S.; RANIERI, V.E.L.; DELITTI, W.B.C. (orgs.). Ambientalização nas instituições de educação superior no Brasil: caminhos trilhados, desafios e possibilidades. São Carlos: EESC/USP. 350 p.

LARRÁN, M.; ANDRADES, J. (2015) Determining factors of environmental education in Spanish universities. International Journal of Sustainability in Higher Education, v. 16, n. 2, p. 251-271. https://doi.org/10.1108/IJSHE-01-2013-0003 
LEITE, E.B.; SOUZA, T.M.F.; FREITAS, J.M.F.; ANDRADE, M.A.; PEREIRA, L.A. (2014) PUC-Minas sustentável: Plano de sustentabilidade ambiental. In: RUSCHEINSKY, A.; GUERRA, A.F.S.; FIGUEIREDO, M.L.; LEME, P.C.S.; RANIERI, V.E.L.; DELITTI, W.B.C. (orgs.). Ambientalização nas instituições de educação superior no Brasil: caminhos trilhados, desafios e possibilidades. São Carlos: EESC/USP. 350 p.

LO, K. (2015) Campus sustainability in Chinese higher education institutions: Focuses, motivations and challenges. International Journal of Sustainability in Higher Education, v. 16, n. 1, p. 34-43. https://doi.org/10.1108/IJSHE-04-2013-0032

LOZANO, R. (2011) The state of sustainability reporting in universities. International Journal of Sustainability in Higher Education, v.12, n. 1, p. 67-78. https://doi.org/10.1108/14676371111098311

MOREIRA, P.G.; VITTA, P.B.D.; PIMENTA, A.C.; MEIRA, A.M.D.; SILVA, A.R.; CAVALHEIRO, C.H.; ZORIGIAN, C.M.; SUDAN, D.C.; LIMA, E.T.; TAVARES, G.A.; COOPER, M.; LEME, P.C.S.; SIMONELLI, S.B.J.; ALBUQUERQUE, V.G.C.; DELITTI, W.B.C. (2014) Construção de política para gestão de resíduos na Universidade de São Paulo como modelo para implementação da PNRS em IES. Revista Eletrônica em Gestão, Educação e Tecnologia Ambiental, v. 18, n. 1, p. 381-387. http://dx.doi.org/10.5902/2236117012435

NICOLAIDES, A. (2006) The implementation of environmental management towards sustainable universities and education for sustainable development as an ethical imperative. International Journal of Sustainability in Higher Education, v. 7, n. 4, p. 414-424. https://doi.org/10.1108/14676370610702217

ROORDA, N. (2001) AISHE: auditing instrument for sustainability in higher education. $\mathrm{DHO}$.

RUSCHEINSKY, A; GOMES, L.P. (2015) Investigação de indicadores de sustentabilidade: trajetória da Unisinos na referência à política dos fluxos da responsabilidade socioambiental. Revista Contrapontos, v. 15, n. 2, p. 261-278.

SACHS, I. (1993) Estratégias de transição para o século XXI. In: BURSZTYN, M. (org.). Para pensar o desenvolvimento sustentável. São Paulo: Brasiliense.

SAVELY, S.M.; CARSON, A.I.; DELCLOS, G.L. (2007) An environmental management system implementation model for US colleges and universities. Journal of Cleaner Production, v. 15, n. 7, p. 660-670. http://doi.org/10.1016/j.jclepro.2006.01.013

SHRIBERG, M. (2002) Institutional assessment tools for sustainability in higher education: strengths, weaknesses, and implications for practice and theory. International Journal of Sustainability in Higher Education, v. 3, n. 3, p. 254-270. https://doi.org/10.1108/14676370210434714

SIBBEL, A. (2009) Pathways towards sustainability through higher education. International Journal of Sustainability in Higher Education, v. 10, n. 1, p. 68-82. https://doi.org/10.1108/14676370910925262

SORRENTINO, M.; BIASOLI, S. (2014) Ambientalização de instituições de educação superior: a educação ambiental contribuindo para a construção de sociedades sustentáveis. In: RUSCHEINSKY, A.; GUERRA, A.F.S.; FIGUEIREDO, M.L.; LEME, P.C.S.; RANIERI, V.E.L.; DELITTI, W.B.C. (orgs.). Ambientalização nas instituições de educação superior no Brasil: caminhos trilhados, desafios e possibilidades. São Carlos: EESC/USP. 350 p.

SORRENTINO, M.; NASCIMENTO, E.; PORTUGAL, S. (2O11) Universidade, educação ambiental e políticas públicas. In: LEME, P.C.S.; PAVESI, A.; ALBA, D.H.; DIAZ, M.J.G. (orgs.). Visões e experiências ibero-americanas de sustentabilidade nas universidades. Granada: Gráficas Alhambra. v. 1. p. 97-102.

TAUCHEN, J.; BRANDLI, L.L. (2006) A gestão ambiental em instituições de ensino superior: modelo para implantação em campus universitário. Gestão \& Produção, v. 13, n. 3, p. 503-515. http://dx.doi.org/10.1590/S0104-530X2006000300012

TAUCHEN, J.A.; BRANDLI, L.L.; FRANDOLOSO, M.A.L. (2010) International net mapping of sustainable universities. In: KNOWLEDGE COLLABORATION \& LEARNING FOR SUSTAINABLE INNOVATION: EUROPEAN ROUNDTABLE ON SUSTAINABLE CONSUMPTION AND PRODUCTION CONFERENCE, 14.; ENVIRONMENTAL MANAGEMENT FOR SUSTAINABLE UNIVERSITIES CONFERENCE, 6., 2010. Anais... Delft.

TOO, L.; BAJRACHARYA, B. (2015) Sustainable campus: Engaging the community in sustainability. International Journal of Sustainability in Higher Education, v. 16, n. 1, p. 57-71. https://doi. org/10.1108/IJSHE-07-2013-0080

UHL, C.; ANDERSON, A. (2001) Green destiny: Universities leading the way to a sustainable future. BioScience, v. 51, n. 1, p. 36-42. https:// doi.org/10.1641/0006-3568(2001)051[0036:GDULTW]2.0.CO;2

VAGNONI, E.; CAVICCHI, C. (2015) An exploratory study of sustainable development at Italian universities. International Journal of Sustainability in Higher Education, v. 16, n. 2, p. 217-236. https://doi.org/10.1108/IJSHE-03-2013-0028

VELAZQUEZ, L.; MUNGUIA, N.; PLATT, A.; TADDEI, J. (2006) Sustainable university: what can be the matter? Journal of Cleaner Production, v. 14, n. 9-11, p. 810-819. http://dx.doi.org/10.1016/j. jclepro.2005.12.008

VELAZQUEZ, L.; MUNGUIA, N.; SANCHEZ, M. (2005) Deterring sustainability in higher education institutions: An appraisal of the factors which influence sustainability in higher education institutions. International Journal of Sustainability in Higher Education, v. 6, n. 4, p. 383-391. https://doi.org/10.1108/14676370510623865

VIEBAHN, P. (2002) An environmental management model for universities: from environmental guidelines to staff involvement. Journal of Cleaner Production, v. 10, n. 1, p. 3-12. https:// doi.org/10.1016/S0959-6526(01)00017-8

WRIGHT, T.S. (2002) Definitions and frameworks for environmental sustainability in higher education. International Journal of Sustainability in Higher Education, v. 3, n. 3, p. 203-220. https://doi. org/10.1108/14676370210434679 